\title{
Taylor series based solution of nonlinear-quadratic ODE systems
}

\author{
V. Sátek ${ }^{*, * *}$ P. Veigend ${ }^{*}$ G. Nečasová* \\ * Brno University of Technology, Faculty of Information Technology, \\ Božetěchova 2, 61266 Brno, Czech Republic, \\ (e-mail: satek@fit.vutbr.cz) \\ ** IT4Innovations, VS̆B Technical University of Ostrava, \\ 17. listopadu 15/2172, 70833 Ostrava-Poruba, Czech Republic
}

Keywords: Continuous systems, Ordinary differential equations, Initial value problems, Taylor series, MATLAB

\section{INTRODUCTION}

The "Modern Taylor Series Method" (MTSM) is the numerical integration method that can numerically solve ordinary differential equations (ODEs). The method calculates terms of the Taylor series recurrently for each integration step. The number of calculated terms is generally different for every step and it depends on a defined accuracy of the calculation. Model implementation of MTSM (TKSL software package, Kunovský (1994)), is limited by maximal number of equations and double accuracy.Therefore the method is currently being tested and reimplemented in MATLAB.

Several papers focus on computer implementation of the Taylor series method in a variable-order and variable-step context (see, for instance, Abad et al. (2015), the TIDES software or in Jorba and Zou (2005)). The reduction of rounding errors Rodríguez and Barrio (2012) and utilization of multiple arithmetic Barrio et al. (2011) improves the applicability of Taylor series based algorithms.

This paper demonstrates that the MTSM, specialized to directly solving nonlinear-quadratic ODE systems, solves non-stiff and in some cases stiff systems very fast (in comparison with MATLAB implementation of explicit and implicit ode solvers) and outperforms standard solvers in the considered benchmark problems. This paper is closely connected with Š́tek et al. (2015) where effective solution of linear ODE systems using MSTM was introduced.

\section{SCHEME FOR QUADRATIC ODES}

In this article, we have focused on effective solution of special case of nonlinear-quadratic systems of ODEs. The nonlinear-quadratic systems of ODEs is any first-order ODE that is quadratic in the unknown function. For such system Taylor series based numerical method can be implemented in very effective way.

The best-known and most accurate method of calculating a new value of a numerical solution of ordinary differential equation $y^{\prime}=f(t, y), y(0)=y_{0}$ is to construct the Taylor series Hairer et al. (1987).
The $n$-th order method uses $n$ Taylor series terms in the explicit form

$$
\begin{aligned}
y_{i+1}= & y_{i}+h f\left(t_{i}, y_{i}\right)+\frac{h^{2}}{2 !} f^{[1]}\left(t_{i}, y_{i}\right)+\cdots \\
& +\frac{h^{n}}{n !} f^{[n-1]}\left(t_{i}, y_{i}\right) .
\end{aligned}
$$

Equation (1) for nonlinear-quadratic systems of ODEs can be rewritten in the form

$$
\boldsymbol{y}^{\prime}=\mathbf{A} \boldsymbol{y}^{2}+\mathbf{B} \boldsymbol{y}_{\boldsymbol{j} \boldsymbol{k}}+\mathbf{C} \boldsymbol{y}+\mathbf{b}
$$

where $\mathbf{A} \in \mathbb{R}^{n e \times n e}$ is the matrix for pure quadratic term, $\mathbf{B} \in \mathbb{R}^{n e \times n e(n e-1) / 2}$ is the matrix for mixed quadratic term, $\mathbf{C} \in \mathbb{R}^{n e \times n e}$ is the Jacobian matrix for linear part of the system and $\mathbf{b} \in \mathbb{R}^{n e}$ is the righthand side for the forces incoming to the system. The unknown function $\boldsymbol{y}^{2}$ represents the vector of multiplications $\left(y_{1} y_{1}, y_{2} y_{2}, \ldots, y_{n e} y_{n e}\right)^{T}$; the unknown function $\boldsymbol{y}_{\boldsymbol{j} \boldsymbol{k}}$ represents the vector of mixed terms multiplications $\left(y_{j_{1}} y_{k_{1}}, y_{j_{2}} y_{k_{2}} \ldots, y_{j_{n e(n e-1) / 2}} y_{k_{n e(n e-1) / 2}}\right)^{T}$. The indices $j, k$ come from combinatorics $C(n e, 2)$ and symbol ne stands for the number of equations in ODE system. For simplification we suppose that the constant matrices $\mathbf{A}, \mathbf{B}, \mathbf{C}$ and the constant vector $\mathbf{b}$ are used in system (2).

Higher derivatives of such systems (2) can be effectively computed in MATLAB software MathWorks (2017) using matrix-vector multiplication, e.g. higher derivative $\boldsymbol{y}^{[p]}$ for pure quadratic term with matrix $\mathbf{A}$ should be expressed as

$$
\boldsymbol{y}^{[p]}=\mathbf{A}\left(\sum_{i=0}^{p-2} \boldsymbol{y}^{[p-1-i]} * \boldsymbol{y}^{[i]}\left(\begin{array}{c}
p-1 \\
i
\end{array}\right)+\boldsymbol{y} . * \boldsymbol{y}^{[p-1]}\right),
$$

where the operation '.*' stands for element-by-element multiplication, i.e. $\boldsymbol{y}^{\left[p_{1}\right]} * \boldsymbol{y}^{\left[p_{2}\right]}$ is vector $\left(y_{1}^{\left[p_{1}\right]} y_{1}^{\left[p_{2}\right]}, \ldots\right.$, $\left.y_{n e}^{\left[p_{1}\right]} y_{n e}^{\left[p_{2}\right]}\right)^{T}$. The binomial coefficients $\left(\begin{array}{c}p-1 \\ i\end{array}\right)$ can be effectively precomputed using Pascal triangle, for more information see pascal function in MATLAB software.

\section{NUMERICAL EXPERIMENTS}

All algorithms are implemented in Matlab 2015a and computations are partially performed on SALOMON supercomputer at IT4Innovations VŠB-TU Ostrava 


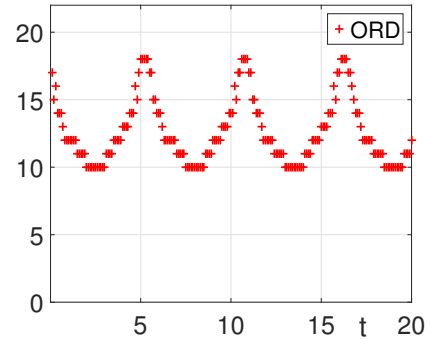

Fig. 1. Order of MTSM for B1 non-stiff problem

IT4Innovations (2017). Relative and absolute tolerance for all computations was set to $10^{-7}$. Classical double precision arithmetic has been used in our examples and maximum order of Taylor series was set to $O R D=60$.

Vectorized MATLAB code of explicit Taylor series expTay with a variable order and variable step size scheme for nonlinear-quadratic systems of ODEs (2) has been implemented. This algorithm was compared on a set of "nonstiff" nonlinear-quadratic systems (see Enright and Pryce (1987)) with vectorized MATLAB explicit ode solvers. Benchmark results are shown in table 1 (each reported runtime is taken as a median value of 100 computations). Ratios of computation times ratio $=$ ode/expTay $>1$ indicate faster computation of the MTSM in all cases (see used orders in Fig. 1).

Table 1. Time of solutions (non-stiff systems): explicit Taylor expTay and MATLAB explicit ode solver comparison

\begin{tabular}{ccccc} 
problem & $\begin{array}{c}\text { ode23 } \\
\text { ratio }\end{array}$ & $\begin{array}{c}\text { ode45 } \\
\text { ratio }\end{array}$ & $\begin{array}{c}\text { ode113 } \\
\text { ratio }\end{array}$ & $\begin{array}{c}\text { expTay } \\
{[\mathrm{s}]}\end{array}$ \\
\hline B1 & 30.67 & 2.05 & 1.57 & 0.0323 \\
B3 & 14.79 & 1.65 & 1.34 & 0.00965 \\
B5 & 29.54 & 2.17 & 1.28 & 0.0201 \\
E4 & 17.1 & 2.12 & 2.19 & 0.00276 \\
\hline
\end{tabular}

The MTSM, due to the higher order, has some positive properties for stability of the solution. Thanks to these properties it can be effectively used for solution of moderately stiff problems. In table 2 one can see the comparisons expTay method with implicit MATLAB ode solvers (see used orders in Fig. 2).

Table 2. Time of solutions (stiff systems): explicit Taylor expTay and MATLAB implicit ode solver comparison

\begin{tabular}{cccccc} 
& $\begin{array}{c}\text { ode15s } \\
\text { problem }\end{array}$ & $\begin{array}{c}\text { ode23s } \\
\text { ratio }\end{array}$ & $\begin{array}{c}\text { ode23t } \\
\text { ratio }\end{array}$ & $\begin{array}{c}\text { ode23tb } \\
\text { ratio }\end{array}$ & $\begin{array}{c}\text { ratio } \\
{[\mathrm{s}]}\end{array}$ \\
\hline C1 & 1.07 & 21.21 & 14.99 & 14.53 & 0.0849 \\
C2 & 1.03 & 20.05 & 14.05 & 13.34 & 0.0823 \\
D1 & 0.23 & 24.75 & 2.9 & 2.33 & 0.569 \\
D3 & 2.12 & 19.55 & 19.4 & 17.28 & 0.065 \\
F3 & 1.2 & 15.31 & 13.14 & 11.75 & 0.0359 \\
\hline
\end{tabular}

\section{CONCLUSION}

The Taylor series scheme (after MATLAB vectorization) seems to be very efficient for solution of some types of nonlinear-quadratic ODEs. In many cases it significantly outperforms standard solvers on the considered benchmark problems.

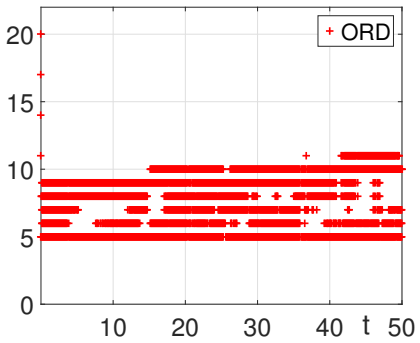

Fig. 2. Order of MTSM for D4 stiff problem

Detailed information and more results will be presented at the conference.

\section{ACKNOWLEDGEMENTS}

This research was financially supported by the Ministry of Education, Youth and Sports from the National Program of Sustainability (NPU II) project IT4Innovations excellence in science LQ1602. The paper also includes the results of the international AKTION research project Number 76p11 and the internal BUT FIT project FIT-S17-4014.

\section{REFERENCES}

Abad, A., Barrio, R., Marco-Buzunariz, M., and Rodríguez, M. (2015). Automatic implementation of the numerical taylor series method: a mathematica and sage approach. Applied Mathematics and Computation, 268, $227-245$.

Barrio, R., Rodríguez, M., Abad, A., and Blesa, F. (2011). Breaking the limits: The taylor series method. Applied Mathematics and Computation, 217, 7940-7954.

Enright, W.H. and Pryce, J.D. (1987). Two fortran packages for assessing initial value methods. In $A C M$ Trans. Math. Softw., volume 13, 1-27. ACM.

Hairer, E., Nørsett, S.P., and Wanner, G. (1987). Solving Ordinary Differential Equations I. vol. Nonstiff Problems. Springer-Verlag Berlin Heidelberg. ISBN 3-54056670-8.

IT4Innovations (2017). "National Supercomputing Center, VŠB-Technical University of Ostrava, Salomon Cluster Documentation - Matlab Overview". URL https://docs.it4i.cz/.

Jorba, A. and Zou, M. (2005). A software package for the numerical integration of ODE by means of high-order Taylor methods. In Exp. Math., volume 14, 99-117.

Kunovský, J. (1994). Modern Taylor Series Method. FEIVUT Brno. Habilitation work.

MathWorks, T. (2017). MATLAB and Simulink software. URL http://www.mathworks.com/products/matlab/ [online].

Rodríguez, M. and Barrio, R. (2012). Reducing rounding errors and achieving brouwers law with taylor series method. Applied Numerical Mathematics, 62, 10141024.

Šátek, V., Kocina, F., Kunovský, J., and Schirrer, A. (2015). Taylor series based solution of linear ode systems and matlab solvers comparison. In MATHMOD VIENNA 2015 - 8th Vienna Conference on Mathematical Modelling, ARGESIM REPORT No. 44, 693-694. ARGE Simulation News. 